\title{
Sequential spectral domain OCT documentation of retinal changes after branch retinal artery occlusion
}

This article was published in the following Dove Press journal:

Clinical Ophthalmology

II April 2010

Number of times this article has been viewed

\section{RK Murthy \\ S Grover \\ KV Chalam}

Department of Ophthalmology, University of Florida-Jacksonville, Jacksonville, FL 32209, USA
Correspondence: KV Chalam

University of Florida-Jacksonville

Department of Ophthalmology 580 West

8th Street Plaza II, 3rd Floor Jacksonville,

Florida 32209 USA

Tel + I 904 244-936I

Fax + I 904 244-939|

Email kchalam@jax.ufl.edu
Abstract: Branch retinal artery occlusions (BRAO) are characterized histopathologically by inner retinal edema initially and atrophy in the presence of persistent ischemia. The duration of ischemia leading to irreversible atrophic retinal changes is not clear. Spectral-domain optical coherence tomography (SD-OCT) provides non-invasive detailed in-vivo histological changes in the retina. In this case report, we show sequential in vivo pathological changes seen in the inner retinal layers, in spite of clinical improvement, following the migration of an intraretinal embolus on the optic nerve head, which had previously resulted in symptomatic BRAO.

Keywords: BRAO, optic nerve head plaque, SD-OCT

\section{Introduction}

Branch retinal artery occlusions (BRAO) are usually embolic in nature. The embolic source is either a carotid artery atheroma or myocardial thrombus. ${ }^{1,2}$ The embolus usually lodges at the bifurcation of the central retinal artery into the branch retinal artery. Histopathologically acute branch retinal artery occlusion reveals ischemia in the corresponding retinal quadrant marked by inner retinal edema in the initial stages and atrophy in long standing cases. ${ }^{3,4}$ Optical coherence tomography (OCT) has been described to show the high reflectivity corresponding to the edematous inner retina layers and hyporeflective signal from the photoreceptor layer. However, the resolution of the images on conventional time-domain OCT is not adequate to resolve the changes in individual layers of the retina. ${ }^{5}$ Spectral-domain OCT (SD-OCT) by its ability to acquire a large number of images in a short period of time provides high resolution cross sectional images of the retina. ${ }^{6}$ Spectralis, one of the commercially available SD-OCT also incorporates TruTrack technology which can image identical areas of the retina at different periods of time, with high point to point correlation.

In this case report we describe the use of Spectralis to document the progression of pathological changes seen in the inner retinal layers, in spite of clinical improvement, following the migration of an intraretinal embolus on the optic nerve head, which had previously resulted in symptomatic BRAO.

\section{Case report}

A 77-year-old white male presented with sudden onset of decreased vision in his right eye of two days duration. Past medical history was significant for well controlled diabetes mellitus. On examination, his best-corrected visual acuity (BCVA) was counting fingers at $3 \mathrm{~m}$ in the right eye. Anterior segment examination was 
unremarkable, except for the presence of a right afferent papillary defect. Fundus evaluation of the right eye revealed the presence of a plaque on the optic nerve head within the inferior branch retinal artery and a sectoral grayish retina along the inferior foveal area (Figure 1). Evaluation with Spectralis OCT revealed an increased thickness of the inner nuclear (IN), inner plexiform (IP) and ganglion cell/nerve fiber (GC/NF) layers in the inferior foveal area (retinal thicknesses of the IN, IP and GC/NF layers were 109, 72 and $74 \mu \mathrm{m}$ in the inferior retina compared to 43,44 and $54 \mu \mathrm{m}$ in the superior retina, Figure 2A). Cross-sectional image through the fovea revealed that the inner segmentouter (IS-OS) line was intact. Sectional image through the optic nerve head revealed a hyperintense area within the inferior branch retinal artery, corresponding to the embolus (Figure 3). A diagnosis of right inferior BRAO was made and the patient was sent for a cardiac evaluation. Work up for giant cell arteritis including ERS and C-reactive protein was within normal range. In the follow up visit after seven days, the patient reported recovery of good visual acuity. BCVA was recorded to be 20/30 in the right eye, with fundus examination noting migration of the embolus peripherally with perfusion of the inferior fovea. A repeat SD-OCT revealed thinning of the IN and IP layers of the inferior retina with preservation of GC/NL layers (retinal thicknesses of the IN, IP and GC/NF layers were 35, 44, and $68 \mu \mathrm{m}$ in the inferior retina, Figure 2B).

\section{Discussion}

Visual prognosis in BRAO is good and $80 \%$ of the patients recover final visual acuity greater than $20 / 40$. However, in the subset of patients who had poor final visual acuity,

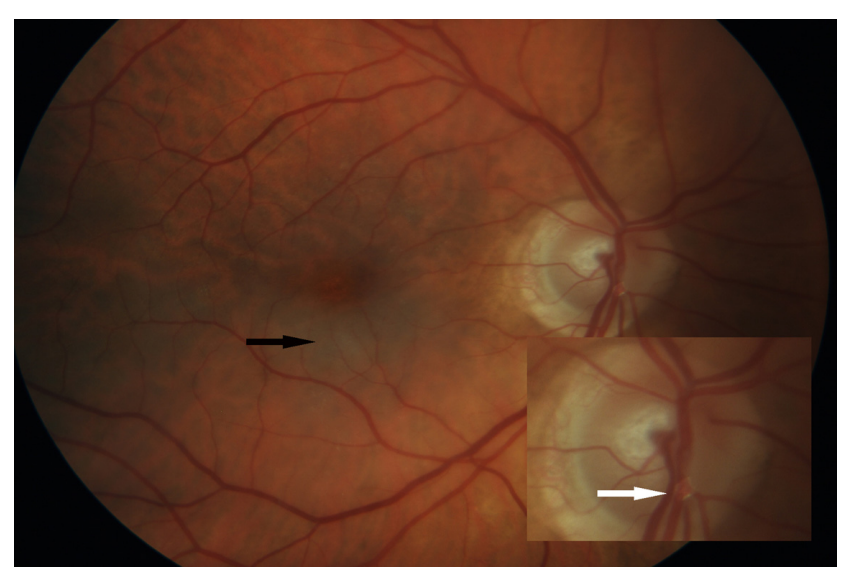

Figure I Fundus photograph of the right eye showing grayish-white edema of the inferior fovea (black arrow). Inset shows enlarged view of the optic nerve head revealing the plaque within the inferior retinal arteriole (white arrow).

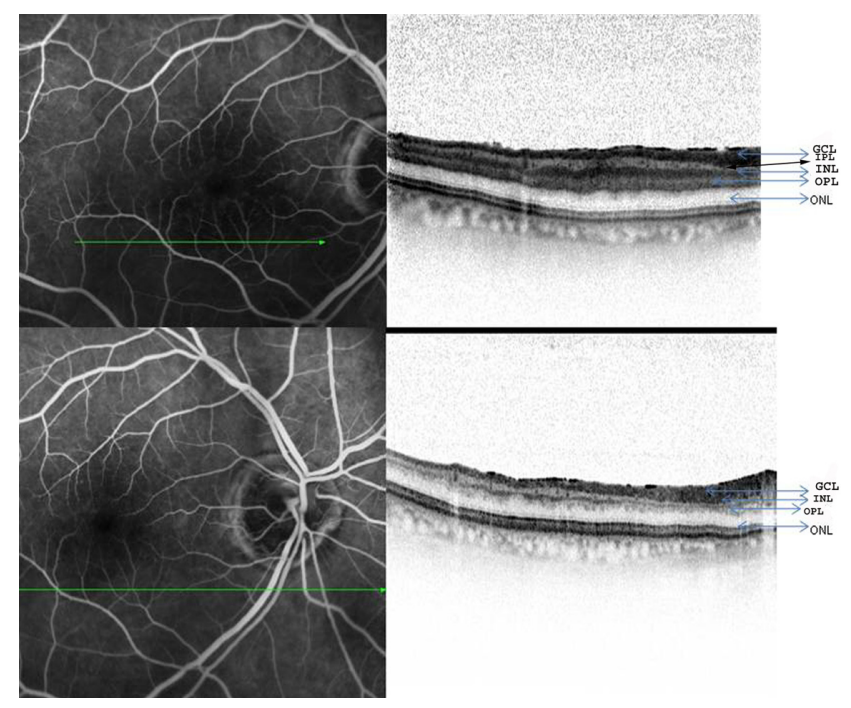

Figure 2 A) Fundus fluorescein and SD-OCT changes of the right eye in a patient after an acute episode of branch retinal artery occlusion revealing edema and thickening of the inner retinal layers (top panel). B) Fundus fluorescein and SD-OCT changes in the same patient after spontaneous resolution of the occlusion following the migration of the embolus (bottom panel).

Abbreviations: ON, outer nuclear layer; INL, inner nuclear layer; IPL, inner plexiform layer and GCL, ganglion cell layer.

Mason et al reported that initial visual acuity of less than 20/100 was the most important negative prognostic factor and advocated aggressive treatment in this subset of patients. ${ }^{1}$

Histologically, early coagulative necrosis of the inner retina with intracellular edema of neurons is seen after a branch retinal arteriole is obstructed. ${ }^{2}$ Hayreh and associates showed that the primate retina underwent irreversible ischemic changes after 105 minutes. ${ }^{3}$ In our patient, on the SD-OCT, the edematous inner retina, comprising of the INL, IPL and GCL was seen as a hyperintense band with increased thickness and could be contrasted with the normal reflectivity and thickness of the corresponding layers in the superior unaffected fovea. Prolonged ischemia results in consecutive atrophy of these layers with each layer exhibiting differential sensitivity to the underlying hypoxia. Animal experiments have revealed retinal ganglion cells to be relatively resistant to the ischemia compared to the other retinal neurons. ${ }^{4}$ Similar finding was observed in our case in vivo by the SD-OCT images which revealed the relative preservation of ganglion cell layer as opposed to the thinning of the inner plexiform and nuclear layers. The images on SD-OCT, Spectralis have the added advantage of being acquired with Tru-track ${ }^{\mathrm{TM}}$ technology, which ensures the imaging of the same area of the retina at different time periods, consistently.

The ability of SD-OCT to delineate the layers of the retina is used in prognosticating visual outcomes in 


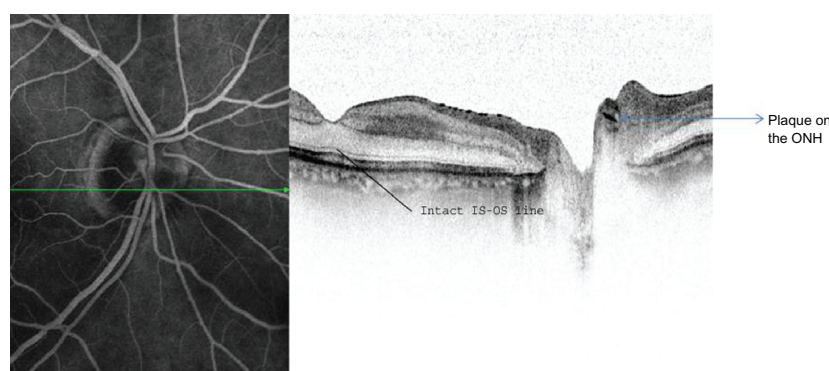

Figure 3 Fundus fluorescein and SD-OCT cross section through the optic nerve head revealing the highly reflective plaque within the lumen of the branch retinal artery. (IS-OS line = Inner segment-Outer segment line).

Abbreviations:

various retinal disorders. Preservation of integrity of the IS-OS line and the external limiting membrane has been reported to have prognostic value in cystoid macular edema due to vascular occlusions. ${ }^{6}$ Presence of an intact IS-OS line at the fovea in our patient suggested the preservation of structural integrity of the photoreceptor layer at the fovea, contributing to the recovery of good visual acuity even in the presence of thinning of the inner nuclear and plexiform layers.

In summary, we report presence of an intact IS-OS line on SD-OCT, which reflects good visual prognosis in a patient with BRAO, despite poor visual acuity at the time of presentation.

\section{Disclosures}

The authors report no conflicts of interest in this work.

\section{References}

1. Mason JO 3rd, Shah AA, Vail RS, Nixon PA, Ready EL, Kimble JA. Branch retinal artery occlusion: visual prognosis. Am J Ophthalmol. 2008;146(3):455-457.

2. Yanoff M, Fine BS. Central retinal artery occlusion. In: Ocular pathology, a text and atlas, 2nd ed. Philadelphia, PA: Harper \& Row. 1982:492-494.

3. Hayreh SS, Jonas JB. Optic disk and retinal nerve fiber layer damage after transient central retinal artery occlusion: an experimental study in rhesus monkeys. Am J Ophthalmol. 2000;129(6):786-795.

4. Goldenberg-Cohen N, Dadon S, Avraham BC, Kramer M, Hasanreisoglu M, Eldar I. Molecular and histological changes following central retinal artery occlusion in a mouse model. Exp Eye Res. 2008;87(4): 327-333.

5. Leung CK, Tham CC, Mohammed S, Li EY, Leung KS, Chan WM, Lam DS. In vivo measurements of macular and nerve fiber layer thickness in retinal arterial occlusion. Eye. 2007;21(12):1464-1468.

6. Yamaike N, Tsujikawa A, Ota M, et al. Three-dimensional imaging of cystoid macular edema in retinal vein occlusion. Ophthalmology. 2008;115(2):355-362.
Clinical Ophthalmology

\section{Publish your work in this journal}

Clinical Ophthalmology is an international, peer-reviewed journal covering all subspecialties within ophthalmology. Key topics include: Optometry; Visual science; Pharmacology and drug therapy in eye diseases; Basic Sciences; Primary and Secondary eye care; Patient Safety and Quality of Care Improvements. This journal is indexed on

Submit your manuscript here: http://www.dovepress.com/clinical-ophthalmology-journal

\section{Dovepress}

PubMed Central and CAS, and is the official journal of The Society of Clinical Ophthalmology (SCO). The manuscript management system is completely online and includes a very quick and fair peer-review system, which is all easy to use. Visit http://www.dovepress.com/ testimonials.php to read real quotes from published authors. 